\title{
Introduction
}

\author{
B. Preneel \\ Katholieke Universiteit Leuven \\ ESAT-COSIC \\ K. Mercierlaan 94, B-3001 Heverlee, Belgium \\ bart.preneel@esat.kuleuven.ac.be
}

\section{An Overview}

This volume contains the proceedings of the second workshop on fast software encryption, which was held at the Katholieke Universiteit Leuven in Belgium from the 14th to the 16th of December 1994. It followed a workshop held the previous year at Cambridge, whose proceedings were published as Lecture Notes in Computer Science, Vol. 809. The workshop was organized in cooperation with the International Association for Cryptologic Research (IACR).

The goal of this series of workshops is to advance our understanding of software algorithms for two cryptographic primitives which require very high speeds, namely encryption algorithms and hash functions. The reason for the interest in software is clear. With the proliferation of personal computers and workstations, everyone will have sufficient computing power on his desk or in his briefcase. On the other hand, performance of networks keeps increasing, and applications such as multimedia make use of this increased bandwidth. However, it is quite unlikely that most users will spend a significant amount of money on hardware solutions to secure their applications. Good software solutions require fast algorithms; unfortunately, for performance reasons many applications use weak solutions such as exoring with a constant string or ciphers which are easy to break.

As long as no fast provably secure solutions are available, we will have to live with ad hoc solutions. Designing secure ad hoc solutions is probably not difficult, but developing solutions which are both fast and secure is a hard problem. Progress in this direction is made by the combined effort of making new proposals on the one hand, and trying to find weaknesses in these proposals on the other hand. In parallel, the generalization of specific attacks leads to the development of a theory which supports both the design and analysis activity. Although some powerful tools have been developed, such as correlation attacks, linear attacks, and differential attacks, we are still very far from a theory on how to design ciphers.

At the workshop, about 30 research contributions were presented on this topic. Almost all authors have found the time to produce a final version, which has been incorporated into this volume after a brief review. As could be expected, fewer proposals for new ciphers appeared than in the first edition. However, this volume contains six proposals, as well as new results on the security of these new proposals. 
For the stream cipher papers, we have the following results: U. Baum and S. Blackburn introduce a generalization of clock control, W.G. Chambers studies random permutations and their application to ciphers, and C. Ding presents a thorough study of cyclotomic sequences. Cryptanalysis is done by R. Anderson, who proposes a new algorithm for cryptanalysis of nonlinear filter generators and breaks the FISH algorithm proposed at last year's workshop and by E. Biham and P.C. Kocher, who demonstrate a practical attack on the PKZIP encryption algorithm. J.Dj. Golić explains how linear cryptanalysis can be extended to stream ciphers. A. Klapper discusses feedback with carry shift registers over certain rings of algebraic integers, and D.J.C. MacKay shows how free energy minimization can be used in the optimization of fast correlation attacks on stream ciphers.

Concrete proposals for new stream ciphers can be found in the contributions of Ross Anderson (PIKE), W.G. Chambers, and J.Dj. Golić.

Three new block ciphers are proposed by R.L. Rivest (RC5), M. Blaze and B. Schneier (McGuffin) and D.J. Wheeler and R.M. Needham (TEA). L.R. Knudsen develops extensions of differential attacks. J.L. Massey discusses differential cryptanalysis of SAFER-K64 (presented at last year's workshop) and presents a variant with a 128-bit key (SAFER-K128). V. Rijmen and B. Preneel show how differential attacks to DES can be extended to hash functions based on DES. The same authors also cryptanalyze the McGuffin algorithm of M. Blaze and B. Schneier. B.S. Kaliski Jr. and M.J.B. Robshaw study the linear cryptanalysis of FEAL using multiple linear relations. U. Blocher and M. Dichtl discuss a problem which occurs in linear cryptanalysis of the DES when more than one Sbox is active per round. S. Vaudenay investigates multipermutations and shows how the absence of this property can be used to cryptanalyze MD4 and a variant of SAFER-K64.

Supporting theory is discussed by H. Dobbertin (bent functions), X. Lai (additive and linear structures), J. Daemen (correlation matrices), K. Nyberg (Sboxes with controllable linearity and differential uniformity) and L. O'Connor (linear approximation tables).

S. Lucks proposes a new NP-hard problem on which hash functions and stream ciphers can be based. M. Roe presents methods to reverse engineer a key escrow device. W. Penzhorn proposes a fast algorithm for homophonic coding based on arithmetic coding. M. Roe gives a list of benchmarks for encryption algorithm and hash functions.

No paper was submitted to the proceedings for the following two presentations: P. Landrock: "Do we need two theories?" (on the relation between block ciphers and hash functions) and J.-J. Quisquater: "Printed documents, images and sounds as messages: new open problems for cryptographic functions."

The next edition of the workshop will be held in Cambridge, U.K. from February 21-23, 1996 under chairmanship of Dieter Gollmann (Royal Holloway, University of London). It will be part of the 6-month research programme on "Computer Security, Cryptology and Coding Theory" at the Isaac Newton Institute for Mathematical Sciences in Cambridge. 


\section{Discussion Sessions}

In this section we try to summarize the issues that came up in the discussions between presentations and in the scheduled discussion sessions.

\subsection{Stream Ciphers Versus Block Ciphers}

Currently there exist a number of block cipher algorithms in the open literature, including CAST, DES, FEAL, GOST 28147, IDEA, LOKI, LUCIFER, RC5, SAFER, TEA, WAKE, etc. DES and Lucifer were designed in the seventies, FEAL in the late eighties, but all the other proposals are less than five years old. The design principles employed in these algorithms serve as examples for the development of new block ciphers and also provide targets for the development of cryptanalytic attacks. System developers can select one of these for a particular application.

However, the situation for stream ciphers is entirely different, since many types of stream ciphers have been proposed and analyzed, but few standardized and published algorithms are available. The SEAL stream cipher described in the proceedings of the 1993 Cambridge workshop is a notable exception. This can in part be explained by the fact that stream ciphers are found mostly in hardware implementations, which often use proprietary algorithms. Especially in environments where error propagation and synchronization are important, specific stream ciphers are much more efficient than the Output FeedBack mode (OFB) or the Cipher FeedBack mode (CFB) of a block cipher.

The current situation may be described as follows: for block ciphers, we have little theory, but many algorithms, while for stream ciphers, we have much theory, but very few completely specified algorithms. It should be noted however that recent developments in cryptanalysis (such as the discovery of differential and linear cryptanalysis) have stimulated the development of theoretical work on block ciphers; these proceedings form a clear witness to this.

It was recognized that a definite need exists for good stream cipher algorithms. More specifically, we need concrete designs for stream ciphers based on existing or new theoretical work in the area, and which may be identified by "catchy" names, as in the case of block ciphers. If these algorithms are placed in the public domain, they could serve as good design examples and also as targets for the development of effective cryptanalytic attacks. This in turn would lead to the establishment of proven design principles for stream ciphers. Also, more stream ciphers would be used in software applications.

\subsection{Criteria for New Algorithms}

One very important observation is that there exists a definite need for fast encryption algorithms and hash functions, designed specifically for software implementation. Older algorithms, like DES, are largely based on bit-oriented operations; therefore straightforward implementations are very slow on generalpurpose computers. It should be noted however that this has not stopped certain groups from developing very fast DES implementations, which are only two 
to three times slower than most new block ciphers (only WAKE is an order of magnitude faster than all the others). Another important consideration is that encryption algorithms implemented in software appear to be less vulnerable to export control restrictions than hardware devices. It was also remarked that cryptographic algorithms are for the time being completely absent in all benchmarks for computers or compilers.

Designers of software algorithms should aim to exploit the parallelism offered by byte/word oriented operations, since shift/rotate operations and integer addition/exor are efficiently performed by most modern computers. Multiplication is also an obvious candidate, but it is slow compared to the other operations (multiplication of two 32-bit registers requires 40 cycles on a 80486 processor). For the design of stream ciphers this would imply the development/extension of the theory of linear feedback shift-registers to the general case of byte/word operations.

It was agreed that look-up tables are a useful building block, but in order to obtain a high speed, the tables should fit into the computer's cache memory. A popular size for the tables seems to be $8 \mathrm{Kbyte}$. An alternative is to use data-dependent operations, following the example set by RC5.

A challenge was put forth (by E. Biham) for anyone to develop an encryption algorithm based on bent functions. In spite of the current development of the theory of bent functions, few cryptographic applications have been presented thus far. In response to this it was mentioned that C. Adams and S. Tavares have proposed the CAST block cipher, which uses S-boxes with 8 input bits and 32 output bits, where the individual output bits are bent functions.

It was concluded that for optimal performance under various circumstances, different algorithms will be required: there is a need for very compact algorithms (for example for smart cards), high speed algorithms (for hardware and for general purpose processors), fast self-synchronizing stream ciphers (for communications applications), and algorithms which lend themselves to implementation in a FPGA (Field Programmable Gate Array).

\subsection{Provable Security}

It was suggested (by J.L. Massey) that we should continue to pursue the development of a theory of provable security. Ideally, we would like to state the equivalence of Shannon's Channel Coding Theorem for cryptography. This would guarantee the existence of provably secure cryptographic systems and provide us with the confidence of pursuing an attainable goal. This pursuit would probably warrant a fresh look at complexity theory. From a cryptographic viewpoint it is important to distinguish between $P / N P$-complexity and Boolean complexity, which is expressed for example, in terms of gate count. This subject has already received some attention in the past and is in dire need of further exploitation. 


\subsection{Hash Functions and Secrecy}

Experience has shown that hash functions are less susceptible to export control restrictions than cryptographic algorithms dedicated to secrecy. It was suggested to consider the development of special modes of hash functions, which are capable of providing secrecy. It was remarked that this requires different properties from the hash functions as those for which hash functions have been designed and evaluated. Also, the use of stream ciphers for authentication should receive further attention.

\section{Acknowledgment}

I would like to thank the participants for their contributions, and for providing the papers in $\mathrm{IAT}_{\mathrm{E}} \mathrm{X}$ format, which has enabled me to edit this volume in electronic format. I also would like to thank the organizing committee:

- Ross Anderson (University Computer Laboratory, Cambridge)

- Eli Biham (Technion, Haifa)

- Cunsheng Ding (University of Turku, Finland)

- Dieter Gollmann (Royal Holloway College, University of London)

- James L. Massey (Swiss Federal Institute of Technology, Zürich)

for the hard work and for the fine collaboration, and Lars R. Knudsen and Kaisa Nyberg for helping with the review of a paper. I am grateful to Walter Penzhorn, who made available his notes on the discussion sessions. Special thanks go to Rita De Wolf and Vincent Rijmen for assisting with the local organization, and to our sponsors Europay International, Microsoft, and Uti-maco Belgium for their generous support. 